'Unidad Docente, Universidad de Chile, Instituto Nacional del Tórax. Santiago, Chile. ${ }^{2}$ Departamento de Enfermedades Respiratorias, Clínica Santa María. Santiago, Chile.

${ }^{3}$ Escuela de Kinesiología, Facultad de Ciencias de la Salud, Universidad de Las Américas. Santiago, Chile. ${ }^{4}$ Escuela de Kinesiología, Facultad de Odontología y Salud, Universidad

Diego Portales. Santiago, Chile. 5Programa de Fisiología y Biofísica, Instituto de Ciencias Biomédicas, Facultad de Medicina, Universidad de Chile, Santiago, Chile.

${ }^{6}$ Departamento de Enfermedades Respiratorias, Hospital Clínico Universidad de Chile, Santiago, Chile.

${ }^{7}$ Escuela de Kinesiología, Facultad Ciencias de la Salud, Universidad San Sebastián. Santiago, Chile.

${ }^{8}$ Dirección de Postgrado, Facultad de Ciencias, Universidad Mayor. Santiago, Chile. aKinesióloga. bioquímico. cDoctor en Ciencias (PhD).

Los autores declaran no tener conflictos de interés. Este trabajo no obtuvo financiamiento.

Recibido el 2 de junio de 2019, Aceptado el 2 de enero de 2020.

Correspondencia a: Astrid von Oetinger Escuela de Kinesiología, Facultad Ciencias de la Salud, Universidad San Sebastián, Lota 2464, Providencia. Santiago, Chile. astridvon@gmail.com

\section{Test cardiopulmonar: una herramienta de utilidad diagnóstica y pronóstica}

\author{
MÓNICA ZAGOLIN ${ }^{1,2}$, LUZ MARÍA TRUJILLO ${ }^{3,4, a}$, \\ SERGIO VILLANUEVA ${ }^{5, b, c}$, MAURICIO RUIZ ${ }^{2,6}$, \\ ASTRID VON OETINGER ${ }^{7,8, a}$
}

\section{Cardiopulmonary exercise test for diagnostic and prognostic purposes}

The functional assessment of patients with dyspnea usually uses static or submaximal exercise tests, which provide limited information because they do not expose patients to the real situation that causes exercise intolerance. The cardiopulmonary exercise test (CPET) is an increasingly used tool that can be used in these circumstances. It determines peak oxygen consumption, anaerobic threshold and cardiac and respiratory reserves, measuring oxygen uptake and carbon dioxide production during standardized exercise conditions. It is useful for risk assessment in cardiothoracic surgery and can provide valuable information such as the timing for transplant in patients with severe chronic disease. The test is non-invasive, has a short duration, and exhibits an adequate safety profile in specialized centers. It is mainly indicated for the dynamic evaluation of athletes or patients with heart, respiratory, and neuromuscular diseases, it is essential part of the study of dyspnea of unknown origin, and in the prognostic assessment of patients who face highly complex interventions. This review provides a comprehensive review of CPET with emphasis on its main indications in healthy people, athletes and, in particular, in functional evaluation of patients with exercise limitations in the context of their chronic diseases.

(Rev Med Chile 2020; 148: 506-517)

Key words: Exercise Test; Oxygen consumption; Physical Functional Performance.
L a disnea es el síntoma principal de muchas enfermedades cardíacas, pulmonares y euromusculares, que se manifiestan con intolerancia al ejercicio. Dentro de la evaluación del paciente con disnea se dispone de exámenes funcionales tales como espirometría, test de difusión con monóxido de carbono, electrocardiograma (ECG) y ecocardiografía, entre otras. Sin embargo, todas son evaluaciones en reposo que no dan cuenta certeramente de la intolerancia al esfuerzo ${ }^{1}$. Otras pruebas que sí son de esfuerzo, tales como el test de caminata de 6 min (TC6m), el test de escalera, el test incremental de la lanzadera ("shuttle test") son evaluaciones submáximas o máximas, pero que no logran precisar el origen de la disnea, ni dan información completa sobre el estado funcional de los diferentes sistemas relacionados con este síntoma ${ }^{2}$.

Por su parte, los bajos niveles de aptitud cardiorrespiratoria (ACR) están asociados a un alto riesgo de enfermedad cardiovascular y mortalidad por todas las causas ${ }^{3}$. La Asociación Americana 
para el corazón (AHA) cataloga a la ACR como un signo clínico vital, ya que es un predictor de mortalidad aún más relevante que los factores de riesgo establecidos (tabaquismo, hipertensión, dislipidemia y diabetes mellitus tipo 2). De acuerdo con ello, la AHA sugiere que la evaluación de la ACR debe ser masificada ${ }^{3}$, siendo la prueba de ejercicio máximo o test cardiopulmonar (CPET) el método estándar para ello ${ }^{3,5}$.

El CPET o ergoespirometría es un examen que permite evaluar de manera integrada, dinámica y precisa la función cardíaca, respiratoria, metabólica y neuromuscular mediante el análisis de gases espirados durante un esfuerzo máximo estandarizado $^{6}$. Esta evaluación permite la medición del consumo máximo de oxígeno $\left(\mathrm{VO}_{2}\right.$ peak $o$ VO$\left.{ }_{2} m a ́ x\right)$, entre otras múltiples variables fisiológicas y parámetros ergoespirométricos ${ }^{7}$.

Históricamente, el CPET fue desarrollado para la evaluación de la capacidad física de deportistas, principalmente aquellos involucrados en disciplinas aeróbicas. En estos casos el $\mathrm{VO}_{2}$ máx no solo predice el rendimiento físico, sino que permite planificar los entrenamientos de acuerdo con la evolución de dicho parámetro, optimizando el desempeño deportivo ${ }^{8}$.

En la actualidad el CPET se ha posicionado como una herramienta determinante en el ámbito clínico, permitiendo la valoración de pacientes con diversas patologías, fundamentalmente del ámbito cardiopulmonar ${ }^{3}$. Se utiliza tanto en la evaluación inicial como posterior a un programa de rehabilitación, o también previo a una intervención quirúrgica a modo de predictor de riesgo ${ }^{6}$. Adicionalmente, ha sido utilizado en la valoración del resultado de terapias y en la categorización de riesgo para trasplantes cardíaco o pulmonar e instalación de dispositivos de asistencia ${ }^{5-8}$.

Al permitir una evaluación dinámica, el CPET reproduce más fielmente los problemas que se presentan en sujetos que tienen algún grado de intolerancia a las actividades de la vida diaria o que presentan disnea con el ejercicio ${ }^{9,10}$.

Recientemente, la AHA recomendó la realización anual de un CPET como práctica clínica de rutina -similar a los exámenes de salud preventiva- considerándolo como la mejor opción en las enfermedades crónicas ${ }^{3}$. En Chile, a pesar de la mayor difusión y disponibilidad del CPET, sigue siendo una herramienta subutilizada; fundamentalmente por el desconocimiento de sus indicaciones y perfil de seguridad, así como por su elevado costo e insuficiente cobertura por los sistemas aseguradores ${ }^{11}$. Es por ello que el objetivo de este artículo es presentar una visión actualizada del CPET como herramienta clínica y destacar la importancia de esta prueba en la evaluación de la ACR.

\section{Principios del test cardiopulmonar}

El CPET es un examen no invasivo, ambulatorio y breve $(<20 \mathrm{~min})^{4}$. Durante la prueba, el sujeto se somete a un esfuerzo incremental de carácter máximo ${ }^{1,2}$, permitiendo evaluar paralelamente parámetros oxihemodinámicos como frecuencia cardiaca $(\mathrm{fc})$, electrocardiograma de 12 derivaciones, presión arterial (PA), oximetría de pulso para medición de saturación $\left(\mathrm{SatO}_{2}\right)$ y gases espirados, a través de mascarilla hermética ${ }^{3}$ (Tabla 1, Figura 1).

La integración de todas las variables permite al sistema otorgar información sobre el consumo de oxígeno obtenido $\left(\mathrm{VO}_{2}\right.$ máx o $\mathrm{VO}_{2}$ peak), equivalente ventilatorio de $\mathrm{CO}_{2}\left(\mathrm{VE} / \mathrm{VCO}_{2}\right)$, umbral anaeróbico (UA), reserva cardiorrespiratoria, pulso de oxígeno, cociente respiratorio (RER) y carga máxima, entre otras ${ }^{3,11-13}$.

El sujeto puede realizar la prueba en cinta rodante (trotadora) o bicicleta ergométrica, dependiendo del perfil del paciente y de los objetivos del estudio. Las ventajas de utilizar cinta rodante son que moviliza mayor masa muscular, es una actividad conocida y permite obtener valores de $\mathrm{VO}_{2}$ máx hasta 6-12\% superiores al del cicloergómetro $^{15}$, siendo más utilizada en deportistas y personas sanas. El cicloergómetro, en tanto, se utiliza preferentemente en evaluación de pacientes, adultos mayores y niños sanos, por razones de seguridad, ya que podrían experimentar inestabilidad o mareo en la trotadora, afectando su rendimiento. Además, este último permite estimar la carga de manera más simple y la monitorización de los parámetros se ve menos afectada por los movimientos de las extremidades ${ }^{3,12}$.

\section{Desarrollo del CPET}

El CPET es un procedimiento seguro (mortalidad: 1 en 10.000) cuando se cumplen las 
Tabla 1. Variables medidas durante la ejecución del CPET

\begin{tabular}{|c|c|c|}
\hline & Generales & $\begin{array}{l}\text { Sintomatología, Test de Borg+, frecuencia cardíaca (Fc), frecuencia } \\
\text { respiratoria, presión arterial, ECG, oximetría de pulso }\end{array}$ \\
\hline \multirow[t]{3}{*}{$\begin{array}{l}\text { Variables } \\
\text { fisiológicas }\end{array}$} & Ventilatorias & $\begin{array}{l}\text { - Reserva ventilatoria } \\
\text { - Ventilación por minuto (VE) } \\
\text { - Volumen corriente (VT) } \\
\text { - Capacidad inspiratoria } \\
\text { - Tiempo inspiratorio/espiratorio }\end{array}$ \\
\hline & Cardiovasculares & $\begin{array}{l}\text { - Reserva cardíaca } \\
\text { - Pulso de oxígeno }\left(\mathrm{VO}_{2} / \mathrm{Fc}\right) \\
\text { - Relación } \mathrm{Fc}_{\mathrm{NOO}}\end{array}$ \\
\hline & Específicas del test & $\begin{array}{l}\text { - } \mathrm{VO}_{2} \text { máx/peak } \\
\text { - Tasa de producción de } \mathrm{CO}_{2}\left(\mathrm{VCO}_{2}\right) \\
\text { - Carga máxima (en watts) } \\
\text { - Cociente respiratorio } \mathrm{VCO}_{2} \mathrm{NO}_{2} \\
\text { - Umbral anaeróbico (UA) } \\
\text { - Valor absoluto y pendiente } \mathrm{VENCO} \text { o equivalente ventilatorio de } \mathrm{CO}_{2} \\
\text { - Presión de oxígeno y de } \mathrm{CO}_{2} \text { al final de la espiración (PETO2 y PET } \mathrm{CO}_{2} \text { ) } \\
\text { - Relación } \mathrm{VO}_{2} \text { /carga }\end{array}$ \\
\hline $\begin{array}{l}\text { Variables ergo- } \\
\text { espirométricas }\end{array}$ & Ventilatorias & $\begin{array}{l}\text { - Ventilación voluntaria máxima (VVM) } \\
\text { - Capacidad vital forzada (CVF) } \\
\text { - Volumen espirado del primer segundo }\left(V E_{1}\right) \\
\text { - Relación VEF, /CVF } \\
\text { - Espacio muerto }\end{array}$ \\
\hline
\end{tabular}

Adaptado de referencias 11-15.

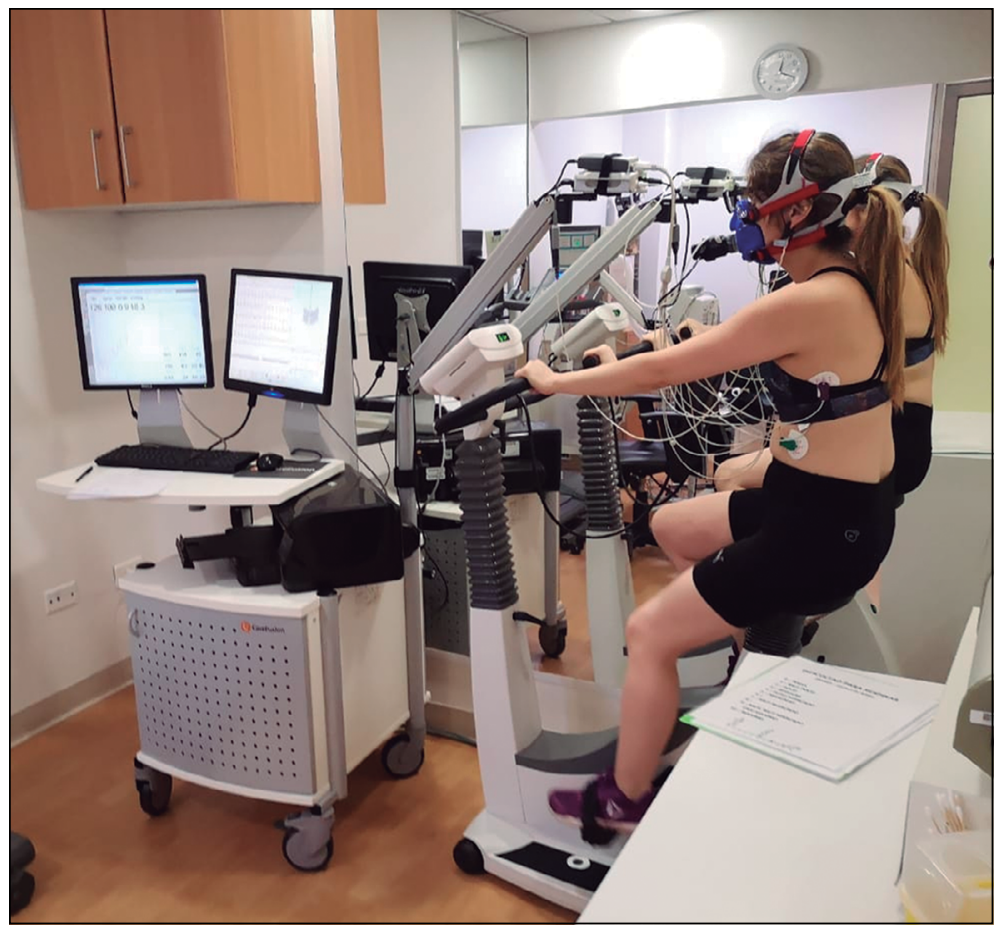

Figura 1. Test cardiopulmonar. Voluntaria sana efectuando el CPET. La mascarilla hermética y los sensores incorporados permiten la determinación y registro en tiempo real de los gases espirados, los que son analizados -en conjunto con otros parámetros fisiológicos- por un software especializado. 
condiciones de ambiente, dotación, monitorización y evaluación sugeridas por las normativas internacionales ${ }^{15}$. Antes de efectuar la prueba es fundamental evaluar las indicaciones y contraindicaciones del examen, las que se detallan en la Tabla 2.

La prueba consta de una primera etapa de “ajustes", en donde se evalúan parámetros en reposo (1-2 min), seguida de una fase de calentamiento (1-3 min, sin carga o carga muy baja), para familiarizar al paciente con el ejercicio. Luego continuar con cargas incrementales según lo programado y finalizar con una fase de recupe- ración (carga mínima por 1-3 min $)^{16}$. En general, la carga por intervalos fluctúa entre 5 y 35 watts, dependiendo de la patología y condición física, y ésta se incrementa cada minuto hasta el máximo tolerado ${ }^{15,17}$. La prueba idealmente es máxima y puede ser detenida por síntomas que se consideren de riesgo (Tabla 3) o cuando el paciente ya no es capaz de alcanzar las revoluciones requeridas, generalmente entre 55-65 por minuto ${ }^{16}$.

Los protocolos de ejercicio más utilizados son el incremental y el de carga constante. El incremental consiste en aumentar progresivamente la carga hasta llegar al límite de tolerancia del

Tabla 2. Contraindicaciones absolutas y relativas para el CPET

\begin{tabular}{|c|c|c|}
\hline & Contraindicaciones absolutas & Contraindicaciones relativas \\
\hline Cardíaca & $\begin{array}{l}\text { Infarto reciente (3-5 días), angina inestable, arrit- } \\
\text { mias no controladas, estenosis aórtica sintomática, } \\
\text { falla cardíaca sintomática, endocarditis, pericarditis, } \\
\text { miocarditis, disección aórtica, bloqueo aurículo- } \\
\text { ventricular severo }\end{array}$ & $\begin{array}{l}\text { Cardiomiopatía hipertrófica, taqui o bradi-arrítmia, } \\
\text { hipertensión arterial grave } \\
\text { Presión sistólica }>200 \mathrm{mmHg} \\
\text { Presión diastólica }>120 \mathrm{mmHg} \text {, enfermedad } \\
\text { valvular moderada, enfermedad coronaria }\end{array}$ \\
\hline Vascular & Embolia pulmonar o infarto & Hipertensión pulmonar severa \\
\hline Respiratoria & $\begin{array}{l}\text { Asma no controlada, insuficiencia respiratoria agu- } \\
\text { da, saturación de oxígeno }<85 \%\end{array}$ & \\
\hline Generales & $\begin{array}{l}\text { Síncope reciente, limitación ortopédica, trombosis, } \\
\text { infección sistémica, epilepsia, insuficiencia hepática, } \\
\text { incapacidad para colaborar }\end{array}$ & $\begin{array}{l}\text { Desequilibrio hidroelectrolítico }\left(\mathrm{K}^{+}, \mathrm{Mg}^{++}\right) \text {, embara- } \\
\text { zo, enfermedad metabólica no controlada (diabetes, } \\
\text { insuficiencia renal), síndrome inmunodeficiencia } \\
\text { adquirida no tratada }\end{array}$ \\
\hline
\end{tabular}

Adaptado de referencia 15.

Tabla 3. Criterios de finalización del CPET por el operador: absolutos y relativos

\section{Criterios absolutos}

El deseo reiterado o aviso del paciente

Dolor torácico, cianosis, palidez, elevación del segmento ST en ECG

Descenso de presión sistólica > 20 mmHg desde el valor más alto previo

Hipertensión arterial > 250/120 mmHg

Arritmias complejas (bloqueo aurículo-ventricular de $2^{\circ}-3^{\circ}$, extrasístole ventricular frecuente, taquicardia ventricular, flutter o fibrilación ventricular)

Desaturación $<80 \%$, síntomas o signos de insuficiencia respiratoria aguda

Síntomas neurológicos (ataxia, mareo o síncope)

\section{Criterios relativos}

Fatiga, cansancio, disnea y claudicación

Taquicardia no severa incluyendo las paroxísticas supraventriculares

Bloqueo de rama que simule taquicardia ventricular

Adaptado de referencias 15 y 16 . 
sujeto $^{13}$, mientras que el de carga constante evalúa el tiempo a la fatiga, utilizando $50-70 \%$ de la carga máxima obtenida en un CPET incremental previo $^{16}$.

\section{Parámetros de utilidad que entrega el CPET}

Algunos de los principales indicadores que se obtienen del CPET son (Tabla 1).

\section{Consumo máximo de $\mathrm{O}_{2}\left(\mathrm{VO}_{2}\right.$ máx: maximal oxygen uptake)}

Cantidad de oxígeno que el organismo utiliza por unidad de tiempo y refleja el nivel metabólico del individuo. En reposo el $\mathrm{VO}_{2}$ es alrededor de $0,25 \mathrm{~L} / \mathrm{min}$ para una persona sana joven y de tamaño promedio. El VO máx da cuenta de la tolerancia al esfuerzo, se relaciona con la intensidad del ejercicio y se mide en deportistas que logran llegar a una meseta del consumo ${ }^{11} \cdot \mathrm{VO}_{2}$ peak: En pacientes lograr un $\mathrm{VO}_{2}$ máximo no siempre es factible, denominándose $\mathrm{VO}_{2}$ peak (oxygen uptake at peak exercise) al $\mathrm{VO}_{2}$ más alto logrado. $\mathrm{El}$ $\mathrm{VO}_{2}$ peak se expresa en $\mathrm{L} / \mathrm{min}, \mathrm{ml} / \mathrm{kg} / \mathrm{min}$ de peso real o como porcentaje del valor predicho (considerando género, edad, raza y talla). El valor en $\mathrm{ml} / \mathrm{min} / \mathrm{kg}$ corresponde al valor más preciso para comparar con estándares normales, entre pacientes, o del individuo consigo mismo en diferentes momentos ${ }^{12}$.Los principios de la determinación de $\mathrm{VO}_{2}$ máx y $\mathrm{VO}_{2}$ peak se muestran en la Figura 2.

\section{Umbral láctico o anaeróbico (UA)}

Es el valor de $\mathrm{VO}_{2}$ en que se produce un incremento en la concentración de ácido láctico plasmático debido al aporte del metabolismo anaeróbico (glucólisis) ${ }^{4}$. Es un indicador objetivo y reproducible de la capacidad funcional, independiente de la motivación del sujeto. La imposibilidad de la mayoría de los pacientes de lograr el $\mathrm{VO}_{2}$ máx determina la valoración de parámetros submáximos, como el UA, para la evaluación funcional ${ }^{11,12}$.

La determinación del UA es útil como indicador para determinar el nivel de aptitud física, la prescripción de ejercicio o para controlar el efecto del entrenamiento físico. El UA delimita el límite superior respecto al rango de intensidades a las que se puede trabajar con predominio aeróbico, mientras que cargas sobre el UA implican un aumento progresivo en la intensidad de trabajo asociado con una disminución progresiva en la tolerancia al ejercicio ${ }^{13,14}$.

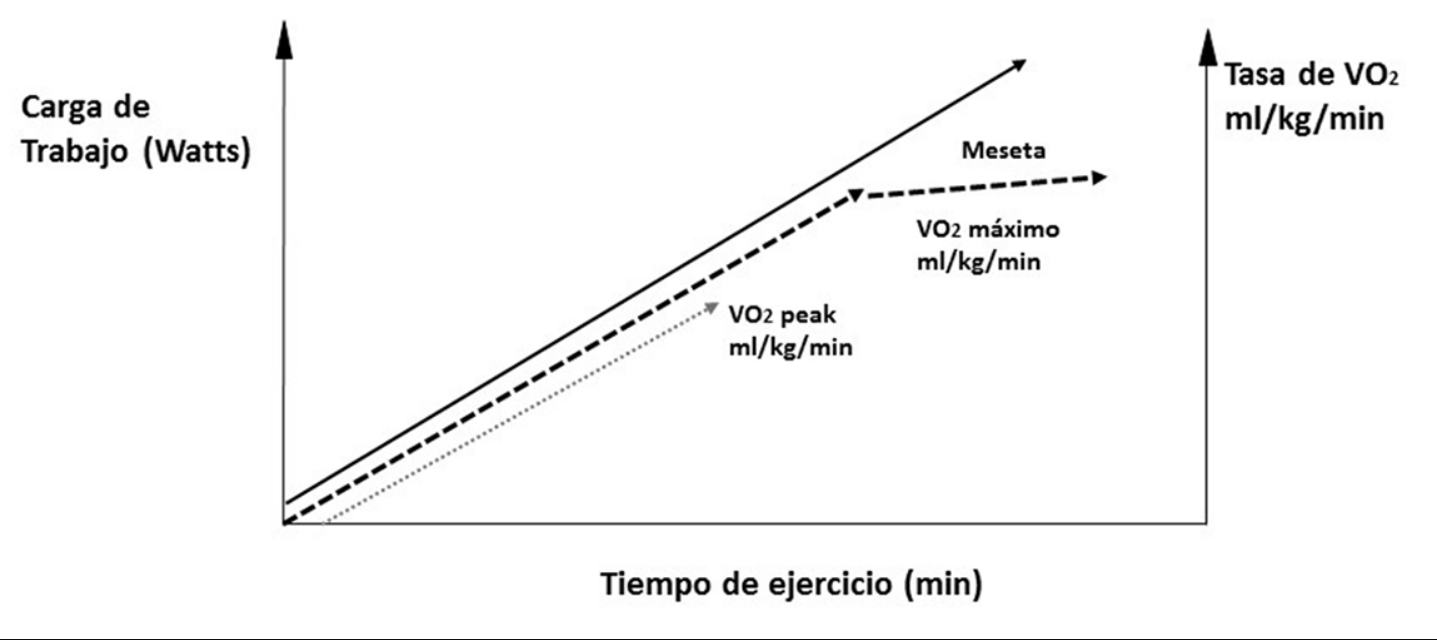

Figura 2. Carga de trabajo y tasa de consumo de oxígeno en el tiempo. La línea continua ilustra la carga de trabajo y las líneas discontinuas muestran la tasa de consumo de oxígeno. La meseta representa el consumo de oxígeno máximo $\left(\mathrm{VO}_{2} \mathrm{max}\right)$ en la línea discontinua gruesa. En individuos que no alcanzan la meseta (línea discontinua delgada) se obtiene el $\mathrm{VO}_{2}$ peak. Adaptado de referencia 15. 


\section{RER (Cociente Respiratorio: $\mathrm{VCO}_{2} / \mathrm{VO}_{2}$ )}

Es la relación entre el volumen de dióxido de carbono producido y el volumen de oxígeno consumido en los tejidos corporales $\left(\mathrm{VCO}_{2} / \mathrm{VO}_{2}\right)$. El RER es un indicador consistente, tanto en sujetos sanos como enfermos y se utiliza para identificar el principal tipo de sustrato energético que se metaboliza durante la respiración celular. Valores RER cercanos a 0,7 se interpretan como oxidación predominante de lípidos y cercanos a 1,0 indican oxidación predominante de carbohidratos, siendo un valor igual o superior a 1,1 indicador de esfuerzo máximo durante el $\mathrm{CPET}^{11,12}$.

\section{Equivalente ventilatorio de $\mathrm{CO}_{2}$}

Es la relación entre la ventilación por minuto (VE) y la eliminación de $\mathrm{CO}_{2}\left(\mathrm{VE} / \mathrm{VCO}_{2}\right)$. En su magnitud inciden el espacio muerto, nivel de perfusión pulmonar y las alteraciones en el patrón ventilatorio. Mientras más elevado es su valor, indica que se requiere mayor número de ventilaciones para eliminar el $\mathrm{CO}_{2}$, traduciendo aumento del espacio muerto por hipoperfusión pulmonar o un trastorno ventilatorio ${ }^{13,14}$.

\section{Relación $\mathrm{VO}_{2} /$ carga de trabajo ( $\mathrm{VO}_{2} / \mathrm{WR}$ )}

Este parámetro representa la eficiencia de conversión de energía metabólica a química para realizar el trabajo mecánico (eficiencia mecánica del sistema musculoesquelético). Una reducción en el valor de esta relación $\mathrm{VO}_{2} / \mathrm{WR}$ indica deficiencias en el transporte de $\mathrm{O}_{2}$, como puede ocurrir con enfermedades del cardiacas, pulmonares o circulatorias, por lo que se recomienda evaluación sistemática de esta pendiente ${ }^{11,15}$.

\section{Recomendaciones de uso de CPET}

Las principales condiciones en las que la utilidad del CPET se encuentra bien fundamentada $^{12-17}$, se resume a continuación, detallándose en la Tabla 4.

\section{Evaluación en deportistas}

Es de gran utilidad para seguimiento de deportistas de alto rendimiento y para los que deseen evaluar de manera objetiva y directa su capacidad aeróbica y nivel de entrenamiento ${ }^{18}$.

\section{Enfermedad cardíaca-respiratoria crónica}

Tanto en evaluación inicial de pacientes como en su seguimiento; en evaluación de nuevas terapias; en la rehabilitación; y en la indicación de trasplante $\mathrm{e}^{16,19}$.

\section{Enfermedad pulmonar obstructiva crónica (EPOC)}

En pacientes sometidos a cirugía resectiva se ha evidenciado una correlación significativa del

Tabla 4. Principales indicaciones del CPET para sujetos sanos para pacientes con alguna patología crónica

\begin{tabular}{|llll|}
\hline Sanos-deportistas & $\begin{array}{l}\text { Paciente con enfermedad } \\
\text { cardíaca }\end{array}$ & $\begin{array}{l}\text { Paciente con enfermedad } \\
\text { respiratoria }\end{array}$ & Otros \\
$\begin{array}{l}\text { Deportistas de alto } \\
\text { rendimiento (capacidad } \\
\text { aeróbica, nivel de entrena- } \\
\text { miento) }\end{array}$ & $\begin{array}{l}\text { Falla cardíaca, eventual uso de } \\
\text { resincronizador }\end{array}$ & $\begin{array}{l}\text { Enfisema, fibrosis, fibrosis quís- } \\
\text { tica, hipertensión pulmonar }\end{array}$ & $\begin{array}{l}\text { Disnea de origen incierto } \\
\begin{array}{l}\text { Sanos previo a programa } \\
\text { de entrenamiento }\end{array}\end{array}$ \\
\hline & Evaluación pre-trasplante & Evaluación pre-trasplante & $\begin{array}{l}\text { Sospecha enfermedad } \\
\text { neuromuscular }\end{array}$ \\
& Rehabilitación & Rehabilitación & $\begin{array}{l}\text { Calificación de incapaci- } \\
\text { dad laboral-pensión de } \\
\text { invalidez }\end{array}$ \\
\hline & $\begin{array}{l}\text { Evaluación de riesgo quirúrgico } \\
\text { (cirugía torácica o abdominal } \\
\text { superior) }\end{array}$ & $\begin{array}{l}\text { Evaluación de riesgo quirúrgi- } \\
\text { co de cirugía torácica-cirugía } \\
\text { resectiva, cirugía reducción de } \\
\text { volumen, cirugía abdominal }\end{array}$ & $\begin{array}{l}\text { Control del peso en en- } \\
\text { fermedades metabólicas }\end{array}$ \\
\hline
\end{tabular}

Adaptado de referencias 12,15,16-19. 
$\mathrm{VO}_{2}$ peak con las complicaciones postoperatorias, así como también entre la pendiente del VE/ $\mathrm{VCO}_{2}$ $>34$ y el riesgo de muerte postoperatoria ${ }^{20-22}$.

\section{Asma}

Tanto en el diagnóstico de asma inducida por ejercicio como en los pacientes asmáticos, el CPET ha mostrado ser útil para la prescripción de programas de ejercicio específicos ${ }^{23}$.

\section{Enfermedad pulmonar difusa}

El CPET ofrece una información más precisa y precoz que el TC6m al momento de prescribir rehabilitación o terapias específicas, así como en el control de estas, o en el momento de enlistar para trasplante ${ }^{24}$.

\section{Hipertensión arterial pulmonar}

El CPET otorga información complementaria a la entregada por el TC6m, ya que el $\mathrm{VO}_{2}$ peak y la pendiente del $\mathrm{VE} / \mathrm{VCO}_{2}$ son predictores independientes de mortalidad ${ }^{25}$.

\section{Obesidad}

Es posible determinar la carga de trabajo que presenta la máxima tasa de oxidación de grasas (Fat $\max$ ), de manera de orientar el nivel de entrenamiento y definir las cargas de trabajo ${ }^{26}$. En los pacientes diabéticos no insulino-dependientes, se ha observado una correlación negativa entre el nivel de glicemia y la capacidad aeróbica, lo que pudiera ser de utilidad en una evaluación más precisa de su condición y en la prescripción de cargas de trabajo en el proceso de rehabilitación con énfasis en la medición del Fatmax ${ }^{27}$.

Por otra parte, la evaluación del $\mathrm{VO}_{2}$ peak es relevante en el seguimiento del tratamiento médico y para la prescripción de cirugía bariátrica. Además, es importante considerar un $\mathrm{VO}_{2}$ peak $\leq 14,5 \mathrm{ml} / \mathrm{kg} / \mathrm{min}$ como indicador pronóstico de complicaciones postoperatorias ${ }^{28,29}$.

\section{Evaluación preoperatoria}

En la evaluación previa a cirugía resectiva pulmonar es fundamental para predecir y minimizar la morbimortalidad perioperatoria y evitar rechazar enfermos oncológicos resecables, permitiendo la evaluación en candidatos a cirugía que presentan espirometría y difusión subnormal ${ }^{30,31}$. El CPET ha permitido distinguir con mayor precisión los pacientes de mayor riesgo quirúrgico
( $\mathrm{VO}_{2}$ peak $<10 \mathrm{ml} / \mathrm{kg} / \mathrm{min} \mathrm{o}<35 \%$ del predictivo $\left.\mathrm{y} \mathrm{VE} / \mathrm{VCO}_{2}>34\right)^{30,31}$.

\section{Otros}

Se utiliza con fines médico-legales y laborales para evaluar incapacidad física parcial o total ${ }^{32}$.

\section{Valor pronóstico del TCP}

El CPET ha demostrado ser útil frente a la necesidad de una intervención, terapia o procedimiento, brindando información pronostica de evolución y sobrevida. De todas las variables que aporta el CPET, el $\mathrm{VO}_{2}$ y el VE/ $\mathrm{VCO}_{2}$ son las que más se han asociado independientemente con el pronóstico, ya que integran aspectos ventilatorios, circulatorios y metabólicos ${ }^{10}$. Su beneficio está demostrado en pacientes con insuficiencia cardiaca, hipertensión pulmonar, cirugía de resección pulmonar, cirugía bariátrica, trasplante renal, EPOC y fibrosis quística ${ }^{7,8,33,34}$.

Los parámetros para utilizar como valor pronóstico acorde a cada condición se detallan en la Tabla 5. A continuación, se detallan algunas patologías en las que el CPET contribuye a la valoración pronóstica.

\section{Insuficiencia cardíaca}

Un $\mathrm{VO}_{2}$ peak $<14 \mathrm{ml} / \mathrm{kg} / \mathrm{min}$ es un punto de corte para referir a una evaluación pretrasplante en pacientes con falla cardíaca, en tanto que aquellos con $\mathrm{VO}_{2}$ peak $>18 \mathrm{ml} / \mathrm{kg} / \mathrm{min}$ muestran una sobrevida igual o mejor que la esperada con trasplante ${ }^{35}$. Se ha reportado que la combinación de $\mathrm{VO}_{2}$ al UA $<11 \mathrm{ml} / \mathrm{kg} / \mathrm{min}$ y un $\mathrm{VE} / \mathrm{VO}_{2}>34$ es un mejor indicador de alto riesgo de mortalidad en falla cardíaca ${ }^{35}$.

\section{Hipertensión pulmonar}

Se he demostrado en pacientes con hipertensión pulmonar que valores de $\mathrm{VO}_{2}<11 \mathrm{ml} / \mathrm{kg} / \mathrm{min}$ con un $\mathrm{VE} / \mathrm{VCO}_{2}>45$ se asocia a una mortalidad de $10 \%$ al año ${ }^{34}$.

\section{EPOC}

Valores de $\mathrm{VO}_{2}$ peak $<10 \mathrm{ml} / \mathrm{kg} / \mathrm{min}$ se ha correlacionado con una mortalidad de $60 \%$ a 5 años $^{33}$. También se ha asociado una reducción significativa de la sobrevida la presencia de un $\mathrm{VE} / \mathrm{VCO}_{2}>34^{36,37}$. 
Tabla 5. Principales indicadores pronósticos según patologia en TCP

\begin{tabular}{|c|c|c|}
\hline Condición de salud & $\begin{array}{l}\text { Variable pronóstica } \\
\text { Punto corte } \mathrm{VO}_{2} \text { peak }(\mathrm{ml} / \mathrm{kg} / \mathrm{min}) \text { o de } \\
\text { equivalente ventilatorio } \mathrm{CO}_{2}\left(\mathrm{VE} / \mathrm{VCO}_{2}\right)\end{array}$ & Interpretación \\
\hline Insuficiencia cardiaca & $\begin{array}{l}\mathrm{VO}_{2}<11 \text { al UA } \\
\mathrm{VO}_{2}<14 \\
{\mathrm{VE} / \mathrm{VCO}_{2}>34}=3\end{array}$ & $\begin{array}{l}\text { Se consideran de alto riesgo de mortalidad } \\
\text { Es un criterio para enlistar para evaluación } \\
\text { de trasplante }\end{array}$ \\
\hline Falla cardíaca & $\begin{array}{l}\mathrm{VO}_{2} \text { al } \mathrm{AT}<11 \\
\mathrm{VE} / \mathrm{NCO}_{2}>34\end{array}$ & Mayor mortalidad \\
\hline Hipertensión pulmonar & $\begin{array}{l}\mathrm{VO}_{2}<11(<35 \%) \\
\mathrm{VE} / \mathrm{VCO}_{2} \text { slope }>45\end{array}$ & $10 \%$ mortalidad anual (HAP1 y HAP2) \\
\hline $\begin{array}{l}\text { Enfermedad pulmonar } \\
\text { obstructiva crónica (EPOC) }\end{array}$ & $\begin{array}{l}\mathrm{VO}_{2}<10 \\
\mathrm{VENCO}_{2}>34\end{array}$ & $\begin{array}{l}\text { Indicadores ambos de mortalidad a cinco } \\
\text { años }\end{array}$ \\
\hline Fibrosis pulmonar Idiopática & $\begin{array}{l}\mathrm{VO}_{2}<13,8 \\
\mathrm{VE} \mathrm{NCO}_{2}>45\end{array}$ & Mayor mortalidad \\
\hline Fibrosis quística & $\begin{array}{l}\mathrm{VO}_{2}<14 \mathrm{ml} / \mathrm{kg} / \mathrm{min} \mathrm{o}<58 \% \text { del valor } \\
\text { predeterminado }\end{array}$ & Indicación para realizar trasplante bipulmonar \\
\hline $\begin{array}{l}\text { Cirugía abdominal, colorrectal, } \\
\text { biliar, urológica, vascular }\end{array}$ & $\mathrm{VO}_{2}<11$ al AT & Se asocia a mayor morbimortalidad \\
\hline \multirow{4}{*}{$\begin{array}{l}\text { Cirugía de resección de } \\
\text { pulmón }\end{array}$} & $\mathrm{VO}_{2}<10$ & Muy elevado riesgo de cirugía resectiva \\
\hline & $\mathrm{VO}_{2}$ entre $10-20$ & $\begin{array}{l}\text { Riesgo moderado. Si se asocia a: } \\
-\mathrm{VE} \mathrm{VCO}_{2}<34 \text { : riesgo bajo } \\
\text { - } \mathrm{VE} / \mathrm{VCO}_{2}>34 \text { : riesgo alto }\end{array}$ \\
\hline & $\mathrm{VO}_{2} \geq 20$ & $\begin{array}{l}\text { Riesgo aceptable para realizar neumonecto- } \\
\text { mía o lobectomía }\end{array}$ \\
\hline & VEN $/ \mathrm{NCO}_{2}>34$ & $\begin{array}{l}\text { Asociación independiente con mortalidad } \\
\text { postoperatoria }\end{array}$ \\
\hline Cirugía bariátrica & $\mathrm{VO}_{2}<14,5$ & $\begin{array}{l}\text { Factor de riesgo mayor para complicaciones } \\
\text { postcirugía }\end{array}$ \\
\hline
\end{tabular}

Adaptado de referencias 3, 5-8, 20, 24, 31, 37.

\section{Fibrosis pulmonar idiopática}

Existe evidencia en pacientes con esta patología que un $\mathrm{VO}_{2}$ peak $\leq 13,8 \mathrm{ml} / \mathrm{kg} / \mathrm{min}$ en conjunto con $\mathrm{VE} / \mathrm{VCO}_{2}>45$ sería indicador de una mayor mortalidad $^{24,38}$.

\section{Evaluación preoperatoria de cirugía resectiva torácica}

El aporte del CPET en la cirugía resectiva torácica, especialmente en cáncer de pulmón, ha sido crucial para estratificar riesgo y predecir complicaciones en el postoperatorio. Se ha consensuado que un $\mathrm{VO}_{2}$ peak $<10 \mathrm{ml} / \mathrm{kg} / \mathrm{min}$ indica un alto riesgo quirúrgico, pudiendo ser un criterio de exclusión del procedimiento; mientras que $\mathrm{VO}_{2}$ peak $>20 \mathrm{ml} / \mathrm{kg} / \mathrm{min}$ sugiere un riesgo estándar, aceptable incluso para neumonectomía ${ }^{30,39-41}$. En los casos intermedios, entre $\mathrm{VO}_{2} 10-20 \mathrm{ml} / \mathrm{k} / \mathrm{min}$, es el $\mathrm{VE} / \mathrm{VCO}_{2}>34$ el que discrimina un mayor riesgo de mortalidad. En casos de riesgo elevado, toma relevancia efectuar una rehabilitación intensiva con niveles de trabajo entre 55-75\% del $\mathrm{VO}_{2}$ peak alcanzado en el estudio basal y se sugiere repetir el CPET ${ }^{40}$ en pocas semanas, esperando una mejor chance quirúrgica $^{39-41}$ (Tabla 5). Al respecto, en los casos de repetición CPET (para cualquier indicación), se estima que una variación inter-ensayo entre 5-10\% del $\mathrm{VO}_{2}$ es aceptable ${ }^{15,42-44}$.

\section{Principios de la interpretación del TCP}

Los resultados de esta prueba dependen de la función de los sistemas que determinan el $\mathrm{VO}_{2}$, 
Tabla 6. Patrones fisiológicos asociados con diferentes diagnósticos

\begin{tabular}{|c|c|c|c|c|c|}
\hline Variable & $\begin{array}{l}\text { Des-acondicio- } \\
\text { namiento }\end{array}$ & $\begin{array}{l}\text { Insuficiencia } \\
\text { cardíaca crónica }\end{array}$ & $\begin{array}{l}\text { Enfermedad } \\
\text { pulmonar }\end{array}$ & $\begin{array}{l}\text { Hipertensión } \\
\text { pulmonar }\end{array}$ & $\begin{array}{l}\text { Enfermedad } \\
\text { mitocondrial }\end{array}$ \\
\hline $\mathrm{VO}_{2}$ peak & $\downarrow$ & $\downarrow$ & $\downarrow$ & $\downarrow$ & $\downarrow$ \\
\hline $\begin{array}{l}\text { Umbral } \\
\text { anaeróbico }\end{array}$ & $\begin{array}{c}\text { Precoz } \\
\left(<30 \% \mathrm{VO}_{2} \text { peak }\right)\end{array}$ & $\begin{array}{c}\text { Precoz } \\
\left(<30 \% \mathrm{VO}_{2} \text { peak }\right)\end{array}$ & $\begin{array}{c}\text { Normal } \\
\left(40-60 \% \mathrm{VO}_{2} \text { peak }\right)\end{array}$ & $\begin{array}{c}\text { Normal } \\
\left(40-60 \% \mathrm{VO}_{2} \text { peak }\right)\end{array}$ & $\begin{array}{c}\text { Normal } \\
\left(40-60 \% \mathrm{VO}_{2} \text { peak }\right)\end{array}$ \\
\hline $\mathrm{VO}_{2} /$ carga & $\begin{array}{c}\text { Normal } \\
\text { (pendiente }=10)\end{array}$ & $\begin{array}{c}\downarrow \\
\text { (pendiente < 10) }\end{array}$ & $\begin{array}{c}\downarrow \\
\text { (pendiente < 10) }\end{array}$ & $\begin{array}{c}\downarrow \\
\text { (pendiente < 10) }\end{array}$ & $\begin{array}{c}\downarrow \\
\text { (pendiente < 10) }\end{array}$ \\
\hline Pulso $\mathrm{O}_{2}$ & Normal & $\downarrow$ & Normal & Normal & Normal \\
\hline VE/VCO ${ }_{2}$ slope & Normal 20-30 & $\uparrow(30-60)$ & $\uparrow(30-60)$ & $\uparrow(>40)$ & Normal \\
\hline $\begin{array}{l}\text { Reserva } \\
\text { ventilatoria }\end{array}$ & Normal & Normal & $\downarrow$ & Normal (o $\downarrow)$ & Normal \\
\hline $\mathrm{SatO}_{2}$ & Normal & Normal & $\downarrow$ & Normal $(\circ \downarrow)$ & Normal \\
\hline PET $\mathrm{CO}_{2}$ & Normal & $\downarrow$ & $\downarrow(30-40 \mathrm{~mm})$ & $\downarrow(30 \mathrm{~mm})$ & Normal \\
\hline VE/NO, slope & Normal & Normal & Normal & Normal & $\uparrow(>100)$ \\
\hline
\end{tabular}

$\downarrow$ : disminuido; $\uparrow$ : aumentado; adaptado de referencias 15,46.

por lo tanto es muy importante reconocer si la limitación tiene origen cardiovascular, respiratorio o de ambos sistemas ${ }^{45,46}$. Para esto deben conocer los patrones fisiológicos asociados a diferentes diagnósticos, comparando los datos resultantes del CPET con los valores de normalidad, resumidos en la Tabla $6^{45,46}$.Además, para una correcta interpretación de la prueba siempre es necesario tener en consideración los puntos a continuación señalados, fundamentales para una correcta valoración clínica y cardiorrespiratoria ${ }^{39,40}$ :

- Motivo de realización del TCP.

- Información clínica del paciente.

- Estado funcional del paciente.

- Causa del término de la prueba.

- Resultados de las diferentes variables en comparación con los valores de referencia (Tabla 7).

Los resultados obtenidos en el CPET permiten planificar estrategias de entrenamiento y elección de las cargas de trabajo (tomando como 100\% el $\mathrm{VO}_{2}$ peak obtenido), así el porcentaje a trabajar dependerá de los objetivos terapéuticos de cada paciente. Las cargas propuestas normalmente son entre $55-75 \%$ del $\mathrm{VO}_{2}$ peak y se recomienda controlar y readecuar cargas cada 2 a 4 semanas, supervisando por un mínimo de 12 semanas para
Tabla 7. Valores normales en el CPET

\begin{tabular}{|ll|}
\hline Variable & Criterio de normalidad \\
$\begin{array}{ll}\mathrm{VO}_{2} \text { peak } \mathrm{V}_{2} \text { max } & >84 \% \text { predictivo } \\
\text { Umbral anaeróbico } & >40 \% \mathrm{VO}_{2} \text { max predicho } \\
& (40-80 \%)\end{array}$ \\
$\begin{array}{l}\text { Frecuencia cardíaca } \\
\text { máxima }\end{array}$ & $>90 \%$ de la predictiva para la \\
edad +
\end{tabular}

*VVmax: ventilación voluntaria máxima; **VD: volumen

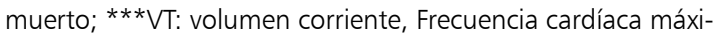
ma: 220-edad. Adaptado de referencia 15.

posteriormente dar paso al entrenamiento autónomo de cada paciente ${ }^{12}$.

Finalmente, es relevante informar si el examen es normal, lo que implica una capacidad aeróbica adecuada; o si es anormal, y -de ser así- aportar 
una aproximación al probable origen de la limitación (cardíaco, respiratorio, muscular o neuropsicológico), dadas las implicancias operativas que ello conlleva ${ }^{45}$.

\section{Conclusión}

Los exámenes estáticos utilizados masivamente hasta la fecha como pruebas de función cardiaca y pulmonar no proporcionan mayor información sobre el rendimiento o eficiencia cardiorrespiratoria, ya que no evalúan de manera dinámica las variaciones de los parámetros fisiológicos asociadas a la generación de disnea, fatiga e intolerancia a la actividad física. En contraste, el CPET es una herramienta efectiva y segura para evaluar la capacidad funcional y la tolerancia al ejercicio, tanto en sujetos sanos como en pacientes con diferentes patologías, especialmente cardiopulmonares, puesto que permite determinar indicadores tales como $\mathrm{VO}_{2}, \mathrm{VE} / \mathrm{VCO}_{2} \mathrm{y} \mathrm{UA}$, sensibles al funcionamiento y adaptación cardiorrespiratoria.

De acuerdo con lo anterior, el CPET es capaz de proporcionar información relevante en un amplio espectro de situaciones, desde la evaluación del rendimiento físico en deportistas hasta la toma de decisiones sobre intervenciones farmacológicas o quirúrgicas en pacientes con patologías crónicas complejas. En consecuencia, los profesionales clínicos de distintas áreas deben tener conocimiento sobre la aplicación, interpretación y utilidad de esta prueba, que en la actualidad se encuentra enormemente subutilizada en relación con la potencialidad diagnóstica, pronóstica y de seguimiento de pacientes que ha demostrado poseer.

Agradecimientos: A las tecnólogas médicas de la Clínica Santa María, por el valioso aporte en la ejecución de cada test, obtención y registro de datos, así como en la asistencia en los controles biológicos.

\section{Referencias}

1. Campbell ML. Dyspnea. Crit Care Nurs Clin North Am 2017; 29 (4): 461-70.

2. Alfonso M, Bustamante V, Cebollero P, Antón M, Herrero S, Gáldiz JB. Assessment of dyspnea and dynamic hyperinflation in male patients with chronic obstructive pulmonary disease during a six minute walk test and an incremental treadmill cardiorespiratory exercise test.Rev Port Pneumol 2017 (5): 266-72.

3. Ross R, Blair SN, Arena R, Church T, Després JP, Franklin B, et al. Importance of assessing cardiorespiratory fitness in clinical practice: A Case for Fitness as a Clinical Vital Sign: A Scientific Statement from the American Heart Association. Circulation 2016; 134: 653-99.

4. Kodama S, Saito K, Tanaka S, Maki M, Yachi Y, Asumi $\mathrm{M}$, et al. Cardiorespiratory fitness as a quantitative predictor of all-Cause mortality and cardiovascular events in healthy men and women: a meta-analysis. JAMA 2009; 301: 2024-35.

5. American College of Cardiology/American Heart Association Task Force on Assessment of Cardiovascular Procedures (Subcommittee on exercise testing). J Am Coll Cardiol 1986; 8: 725-38.

6. Kaminsky L, Imboden M, Arenas R, Myers J. Reference Standards for Cardiorespiratory Fitness Measured With Cardiopulmonary Exercise Testing Using Cycle Ergometry: Data From the Fitness Registry and the Importance of Exercise National Database (FRIEND) Registry. Mayo Clin Proc 2017; 92 (2): 228-33.

7. Balady GJ, Arena R, Sietsema K, Myers J, Coke L, Fletcher GF, et al. Clinician's Guide to cardiopulmonary exercise testing in adults: a scientific statement from the American Heart Association. Circulation 2010; 122: 191-225.

8. Palange P, Ward SA, Carlsen KH, Casaburi R, Gallagher CG, Gosselink R, et al. Recommendations on the use of exercise testing in clinical practice. Eur Respir J 2007; 29: 185-209.

9. Myers J. New American Heart Association/American College of Cardiology guidelines on cardiovascular risk: when will fitness get the recognition it deserves? Mayo Clin Proc 2014; 89: 722-6.

10. Loprinzi PD. Estimated Cardiorespiratory Fitness Assessment as a Patient Vital Sign. Mayo Clin Proc 2018; 93: 821-3.

11. Zagolin M. Ejercicio y pulmón" ¿Qué hemos aprendido en las jornadas de otoño 2012? Rev Chil Enferm Respir 2012; 28 No 2: 87-93.

12. Wasserman K, Hansen C, Stringer W, Sietsema K, Sun C, Whipp B. Principles of exercise testing and interpretation. Philadelphia: Lippincott Williams \& Wilkins; 2011.

13. Arena R, Myers J, Aslam SS, Varughese E, Peberdy MA. Peak $\mathrm{VO}_{2}$ and $\mathrm{VE} / \mathrm{VCO}_{2}$ slope in patients with heart failure: a prognostic comparison. Am Heart J 2004; 147: 354-60. 
14. Mehani SHM, Abdeen HAA. Cardiopulmonary rehabilitation program impact on prognostic markers in selected patients with resting and exercise-induced ventilatory inefficiency: a clinical trial. J Phys Ther Sci 2017; 29 (10): 1803-10.

15. American Thoracic Society/ American College of Chest Physicians ATS/ACCP Statement on Cardiopulmonary Exercise Testing. Am J Respir Crit Care Med 2003; 167: 211-77.

16. Mezzani A, Agostoni $\mathrm{P}$, Cohen-Solal A, Corrà U, Jegier A, Kouidi E, et al. Standards for the use of cardiopulmonary exercise testing for the functional evaluation of cardiac patients: a report from the Exercise Physiology Section of the European Association for Cardiovascular Prevention and Rehabilitation. Eur J Cardiovasc Prev Rehabil 2009; 16 (3): 249-67.

17. Arós F, Boraita A, Alegría E, Alonso A, Bardají A, Lamiel R. Guías de práctica clínica de la Sociedad Española de Cardiología en pruebas de esfuerzo. Rev Esp Cardiol 2000; 53: 1063-94.

18. Herdy AH, Ritt LE, Stein R, Araújo CG, Milani M, Meneghelo RS, et al. Cardiopulmonary Exercise Test: Background, Applicability and Interpretation. Arq Bras Cardiol 2016; 107 (5): 467-81.

19. Brunelli A, Kim AW, Berger KI, Addrizzo-Harris DJ. Physiologic evaluation of the patient with lung cancer being considered for resectional surgery: Diagnosis and management of lung cancer, 3rd ed: American College of Chest Physicians evidence-based clinical practice guidelines. Chest 2013; 143 (5): 166-90.

20. Torchio R, Guglielmo M, Giardino R, Ardissone F, Ciacco C, Gulotta C, et al. Exercise ventilatory inefficiency and mortality in patients with chronic obstructive pulmonary disease undergoing surgery for non-small-cell lung cancer. Eur J of Cardiothorac Surg 2010; 38 (1): 14-20.

21. Brunelli A, Charloux A, Bolliger CT, Rocco G, Sculier JP, Varela G, et al. The European Respiratory Society and European Society of Thoracic Surgeons clinical guidelines for evaluating fitness for radical treatment (surgery and chemoradiotherapy) in patients with lung cancer. Eur J Cardiothorac Surg 2009; 36: 181-4.

22. Shafiek H, Valera JL, Togores B, Torrecilla JA, Sauleda J, Cosío BG. Risk of postoperative complications in chronic obstructive lung disease patients considered fit for lung cancer surgery: beyond oxygen consumption. Eur J Cardiothorac Surg 2016; 50 (4): 772-9.

23. Joyner BL, Fiorino EK, Matta-Arroyo E, Needleman JP. Cardiopulmonary exercise testing in children and adolescents with asthma who report symptoms of exercise-induced bronchoconstriction. J Asthma 2006; 43
(9): 675-8.

24. Van der Plas MN, van Kan C, Blumenthal J, Jansen H, Wells A, Brasser P. Pulmonary vascular limitation to exercise and survival in idiopathic pulmonary fibrosis. Respirology 2014; 19: 269-75.

25. Galiè N, Humbert M, Vachiery JL, Gibbs S, Lang I, Torbicki A, et al. 2015 ESC/ERS Guidelines for the diagnosis and treatment of pulmonary hypertension. Eur heart J 2016; 37: 67-119.

26. Dandanell S, Præst CB, Søndergård SD, Skovborg C, Dela F, Larsen $S$, et al. Determination of the exercise intensity that elicits maximal fat oxidation in individuals with obesity. Appl Physiol Nutr Metab 2017; 42 (4): 405-12.

27. Ucok K, Yalcinkaya H, Acay A, Coban NF, Aslanalp S, Akkan G, et al. Do patients with newly diagnosed type 2 diabetes have impaired physical fitness, and energy expenditures? Neth J Med. 2015; 73 (6): 276-83.

28. Hennis PJ, Meale PM, Hurst RA, O'Doherty AF, Otto J, Kuper M, et al.Cardiopulmonary exercise testing predicts post-operative outcome in patients undergoing gastric bypass surgery. BJA: Br J of Anaesth 2012; 109 (4): 566-71.

29. Moran J, Wilson F, Guinan E, McCormick P, Hussey J, Moriarty J. Role of cardiopulmonary exercise testing as a risk-assessment method in patients undergoing intra-abdominal surgery: a systematic review. Br J Anaesth 2016; 116 (2): 177-91.

30. Harvie D, Levett D. Exercise testing for pre-operative evaluation. Chapter 14-ERS monograph 2018: 251-79.

31. Brunelli A, Belardinelli R, Refai M, Salati M, Socci L, Pompili C, et al. Peak oxygen consumption during cardiopulmonary exercise test improve risk stratification in candidates to mayor lung resection. Chest 2009; 135 (5): 1260-67.

32. Sue DY. Exercise testing in the evaluation of impairment and disability. Clin Chest Med 1994; 15: 369-87.

33. Chakkera HA, Angadi SS, Heilman R, Kaplan B, Scott $\mathrm{RL}$, Bollempalli $\mathrm{H}$, et al. Cardiorespiratory Fitness (Peak Oxygen Uptake): Safe and Effective Measure for Cardiovascular Screening Before Kidney Transplant. J Am Heart Assoc 2018; 7 (11). DOI: 10.1161/ JAHA.118.008662.

34. Wensel R, Opitz C, Anker S, Winkler J, Höffken G, Kleber F, et al. Assessment of survival in patients with primary pulmonary hypertension: importance of cardiopulmonary exercise testing. Circulation 2002; 106: 319-24.

35. Gitt AK, Wasserman K, Kilowski C, Kleemann T, Kilkowski A, Bangert M, Schneider S, Schwarz A, Senges J. Exercise anaerobic threshold and ventilatory efficiency 
identify heart failure patients for high risk of early death. Circulation 2002; 106: 3079-84.

36. Hiraga T, Maekura R, Okuda T, Okamoto T, Hirotani A, Kitada S, Yoshimura K, Yokota S, Masami Ito M, Ogura T. Prognostic predictors for survival in patients with COPD using cardiopulmonary exercise testing. Clin Physiol Funct Imaging 2003; 23: 324-31

37. Neder JA, Alharbi A, Berton DC, Alencar MC, Arbex F, Hirai D. Exercise ventilatory inefficiency adds to lung function in predicting mortality in COPD. COPD 2016; 13: 416-24.

38. Vainshelboim B, Oliverira J, Fox BD, Weiss I, Fox BD, Fruchter $\mathrm{O}$, et al. Theprognostic role of ventilatory inefficiency and exercise capacity in idiopathic pulmonary fibrosis. Respir Care 2016; 61: 1100-109.

39. Mehra M, Canter C, Hannan M, Semigran M, Uber P, David P, et al. The 2016 International Society for Heart Lung Transplantation Listing criteria for heart transplantation: a 10 year update. J Heart Lung Transplant Off Publ Int Soc Heart Transplant 2016; 35 (1): 1-23.

40. Salati M, Brunelli A. Risk Stratification in Lung Resection. Curr Surg Rep 2016; 37: 5-9.

41. Quanjer PH, Tammeling GJ, Cotes JE, Pedersen OF,
Peslin R, Yernault JC. Lung volumes and forced ventilatory flows. Report working party: standardization of lung function testing. Eur Respir J Suppl 1993; 6: 5-40.

42. Tuyet Le VD. Cardiopulmonary Exercise Testing in Aortic Stenosis. Dan Med J 2017; 64 (5): B5352.

43. Decato T, Bradley S, Wilson E, Hegewald M. Repeatability and Meaningful Change of CPET Parameters in Healthy Subjects. Med Sci Sports Exerc 2018; 50 (3): 589-95.

44. Bensimhon D, Leifer E, Ellis S,Fleg J, Keteyian S, Piña I, et al. Reproducibility of Peak Oxygen Uptake and Other Cardiopulmonary Exercise Testing Parameters in Patients with Heart Failure (From the Heart Failure and A Controlled Trial Investigating Outcomes of exercise training). Am J Cardiol 2008; 102 (6): 712-17.

45. Forman DE, Myers J, Lavie CJ, Guazzi M, Celli B, Arena R. Cardiopulmonary Exercise Testing: relevant but underused. Post grad Med 2010; 122 (6): 68-86.

46. Cid-Juárez S, Reyes J, Cortés-Télles A, Gochicoa-Rangel A, Mora-Romero U, Silva-Cerón M, Torre-Bouscoulet L. Prueba cardiopulmonar de ejercicio. Recomendaciones y procedimiento. Neumol Cir Torax 2015; 74 (3); 207-21. 